\title{
Neue pharmakologische Therapieoptionen der idiopathischen Lungenfibrose?
}

\author{
New Treatment Options for Idiopathic Pulmonary Fibrosis?
}

Autoren

Institut
K. Gutjahr, A. Gillissen

Robert-Koch-Klinik, Thoraxzentrum des Klinikums St. Georg, Leipzig eingereicht 1.4.2009

akzeptiert nach Revision 30. 6.2009

Bibliografie

DOI $10.1055 / \mathrm{s}-0029-1214953$

Online-Publikation: 25. 8. 2009

Pneumologie 2009; 63:

512-518 @ Georg Thieme

Verlag KG Stuttgart · New York ISSN 0934-8387

Korrespondenzadresse Dr. Kathleen Gutjahr

Robert-Koch-Klinik

Thoraxzentrum des Klinikums

St. Georg

Nikolai-Rumjanzew-Str. 100

04207 Leipzig

kathleen.gutjahr@sanktgeorg.de

\section{Zusammenfassung}

$\nabla$

Die idiopathische Lungenfibrose (IPF) ist eine chronische Erkrankung des Lungengewebes, die typischerweise progredient verläuft. Auf zellulärer Ebene führt ein Übermaß an profibrotischer und proinflammatorischer Zellaktivität einschließlich einer hohen Last an reaktiven Sauerstoffspezies zu einer Zerstörung des Lungengewebes mit Ausbildung der für das Spätstadium typischen Wabenlunge. Es liegt daher nahe, mittels effektiver Therapiemaßnahmen zu versuchen, diese pathophysiologischen Vorgänge zu blockieren. In dieser Übersicht werden neuere Therapieansätze, wie z.B. die Inhibition von Zytokinen, Eicosanoiden oder Oxidantien, die klinische Wirksamkeit von antifibrotisch oder antikoagulativ wirksamen Medikamenten, vorgestellt. Klinische IPF-Studien haben mit wesentlichen Problemen zu kämpfen. Trotz der in Studien der letzten 5 Jahre umgesetzten genaueren Definition der eingeschlossenen Patientenkollektive (alle nach ATS/ETS-Kriterien definiert) bleibt das Problem der großen Variationsbreite des Krankheitsverlaufs, wobei Subgruppen mitunter einen akzelerierten Verlauf nehmen und andere über einen längeren Zeitraum stabil bleiben. Bislang gibt es keinen Parameter, der eine Vorhersage hinsichtlich des Verlaufs treffen könnte. Dies ist nur durch prospektive Funktionsuntersuchungen möglich. Wenn ein hoher Anteil an neu diagnostizierten Patienten in Studien aufgenommen wird, ist es nicht möglich, nach diesem Aspekt zu stratifizieren. Daher verwundert nicht, dass die meisten neueren Studien das Erreichen ihres jeweils definierten primären Studienendpunktes verfehlten. Nur in Subgruppen- oder post-hoc-Analysen zeigen sich bei einigen sekundären Endpunkten statistisch signifikante Unterschiede, die jedoch nach derzeitigem Kenntnisstand keine Änderung der bisherigen Therapieempfehlungen notwendig machen.

\section{Abstract \\ $\nabla$}

Idiopathic pulmonary fibrosis (IPF) is a chronic condition of unknown etiology with an life-limiting outcome. An excess of profibrotic and proinflammatory mediators as well as reactive oxygen species, resulting in progressive fixed tissue fibrosis, architectural distortion and loss of lung function making it plausible to inhibit these processes therapeutically. In this review new treatment options are discussed including substances with antiinflammatory properties which inhibit cytokines, eicosanoids or oxidants, drugs with antifibrotic efficacy as well as anticoagulative compounds. In the last 5 years treatment trials include only IPF patients characterized by the criteria of the American Thoracic Society and the European Respiratory Society. However, lack of validated outcome measures in most trials representing either disease improvement or progression, and/or sufficient large patient number are still hindering this kind of studies. Unfortunately most studies still failed to meet their primary end-points. Marginal trends or statistically significant differences between treatment groups were only apparent in subgroups or exploratory end-points of post-hoc analysis. Regardless of numerous trails published in recent years and per se promising new drugs, a change in current recommendations in the management of IPF is not warranted at present. 


\section{Einleitung}

\section{$\nabla$}

Die idiopathische Lungenfibrose (IPF) ist nach der Sarkoidose die häufigste Lungenparenchymerkrankung mit einer Prävalenz von ca. 30 pro 100000 bei Männern und 15 pro 10000 bei Frauen. Die Prävalenz steigt mit höherem Lebensalter und liegt bei über 75Jährigen bei 250 pro 100000 [1,2]. Der Erkrankungsverlauf ist häufig progredient und therapeutisch kaum beeinflussbar [3]. Ging man früher davon aus, dass eine chronische Entzündung im Alveolar- und interstitiellen Raum die Ursache der IPF sei, so unterstützen die heutigen Konzepte zur Pathogenese der Fibrose die Hypothese, dass die IPF die Folge einer pathologischen Wundheilung ist. Im Vordergrund stehen dabei wiederholte Epithelschädigungen, die ein gestörtes Remodeling zur Folge haben. Ein wesentlicher pathogenetischer Faktor scheint die Dysregulation auf Ebene der Zytokine zu sein, da über diesen Botenstoff ein Großteil der interzellulären Kommunikation abläuft. Insbesondere dem „transforming growth factor“ (TGF)- $\beta$ wird dabei eine Schlüsselstellung zugeschrieben. Somit wäre die entzündliche Komponente bei der IPF nur eine sekundäre Erscheinung. Im Vordergrund stehen vor allem das pathologische Verhalten von Fibroblasten, Myofibroblasten und Epithelzellen. Diese Hypothese würde auch das ungenügende Ansprechen von Steroiden und Immunsuppressiva erklären.

Vor dem Hintergrund der sehr unbefriedigenden bisherigen Therapieergebnisse dieser aggressiv verlaufenden Erkrankung bleibt nur die Hoffnung, dass ein größeres Wissen über die Pathogenese die Entwicklung wirksamerer Therapeutika stimuliert [4-6]. In dieser Übersicht werden exemplarisch sieben neuere klinische IPF-Studien vorgestellt, in denen pharmakologische Alternativen auf ihre Wirksamkeit evaluiert wurden.

\section{Therapieansätze}

$\nabla$

Folgende pharmakologische Therapien wurden bei der IPF evaluiert:

- Antientzündlich oder immunsuppressiv wirkende Substanzen: Azathioprin/Prednisolon, Pirfenidon, BIBF 1120 (Tyrosinkinase-Inhibitor gegen PDGF, VEGF, FGT), Antikörper gegen Tumornekrosefaktor alpha [TNF $\alpha]$ und TNF $\alpha$-Rezeptoren, Interleukin-13, TGF $\beta$ und MCP-1), Interferon (IFN)-gamma/ -alpha, Thalidomid, Tetrathiomolybdate, N-Acetylcystein [7-12]

- Pharmaka mit einer antifibroproliferativen Wirkung, die gleichzeitig den pulmonal-arteriellen Druck senken [13,14]

- Sonstige: Antikoagulantien [15]

- Nicht-pharmakologische Ansätze, wie z.B. Rehabilitation [16] Bis auf die IFIGENIA- Studie haben die Untersuchungen ihren primären Endpunkt nicht erreicht. Häufig handelt es sich auch nur um Studien mit sehr kleinen Patienten- und Kontrollkollektiven, oder es sind offene Beobachtungsstudien ohne Randomisierung und Placebovergleich.

Bei der IPF handelt es sich um eine sehr schwer pharmakologisch studierbare Erkrankung. Der Grund liegt in der Erkrankungscharakteristik, der Definition der Erkrankung entsprechend der gewählten diagnostischen Kriterien (insbesondere Klinik vs. Lungenbiopsie, vs. Ergebnis der radiologisch-morphologischen Diagnostik), dem gewählten Einschluss und Erfolgskriterien [17]. Ob der 6-Minuten-Gehtest, wie er bei den PAH (pulmonal-arterielle Hypertonie)-Studien als primärer Endpunkt üblich ist, bei IPFPatienten sinnvoll erscheint, bleibt zu hinterfragen. Sinnvolle
Endpunkte erscheinen bei der IPF entsprechend der aktuellen Studienlage wohl eher das progressionsfreie Überleben, die Lungenfunktion (insbesondere die Vitalkapazität) und krankenhauspflichtige Exazerbationen zu sein. Derartige Subgruppen- und post-hoc-Analysen sind sinnvoll, um neue Hypothesen zur Wirksamkeit eines bestimmten Medikamentes zu generieren und darauf aufbauend, weitere Therapiestudien mit modifiziertem Studiendesign und eventuell anderen Endpunkten zu konzipieren. Von herausragender Bedeutung ist neben dem Nachweis der therapeutischen Wirksamkeit die Untersuchung des Sicherheitsaspektes der z.T. auch nebenwirkungsreichen Medikation. Die Fülle der aktuell registrierten Studien (siehe $\bullet$ Tab. 1 und $\bullet 2$ ) zeigt, dass verschiedene Substanzen zur Verfügung stehen, die eventuell die derzeit sehr limitierte Pharmakotherapie wirkungsvoll erweitern könnten.

\section{N-Acetylcystein}

IFIGENIA-Studie (Idiopathic Pulmonary Fibrosis International Group Exploring NAC I Annual)

Bei der IFIGENIA-Studie handelt es sich um eine multinationale, doppelblinde, randomisierte, placebokontrollierte Studie, in die 184 IPF-Patienten eingeschlossen wurden ( Tab. 1). Es wurde über einen Zeitraum von 12 Monaten die Effektivität von N-Acetylcystein (NAC) zuzüglich einer Prednisolon- und AzathioprinTherapie gegenüber Placebo getestet. Als primäre Endpunkte der Studie wurden die Änderungen der Lungenfunktionsparameter Vitalkapazität (VC) und Diffusionskapazität (DLCO) bestimmt. Es zeigte sich ein statistisch signifikant geringerer Abfall der VC und der DLCO in der Verumgruppe im Vergleich zu Placebo. Dieses erstmalig positive Studienergebnis wird durch positive Trends in den sekundären Studienendpunkten, einschließlich Belastbarkeit und dem Überleben der Patienten, untermauert. Ein signifikanter Überlebensvorteil konnte in dieser Studie jedoch nicht nachgewiesen werden. Allerdings müssen hierbei die geringe Fallzahl und Studiendauer berücksichtigt werden. Es wäre denkbar, dass eine derartige Verlangsamung des Abfalls der VC sich auf einen Überlebensvorteil auswirken könnte, da es sich gezeigt hat, dass eine Abnahme der VC um 10\% mit einem signifikant erhöhten Mortalitätsrisiko verbunden ist. Da die IPF zu den seltenen Erkrankungen gehört, besteht immer das Problem, genügend Patienten für eine Studie zu aquirieren bzw. aufgrund der kurzen Überlebensdauer einen ausreichend langen Beobachtungszeitraum zu haben. Deshalb ist bei derartigen Studien, ausschließlich das Überleben als primären Endpunkt zu sehen, kritisch zu beachten. Um Studien bei derart seltenen Erkrankungen überhaupt praktikabel zu machen, sollten in Zulassungsstudien auch Surrogatmarker wie Lungenfunktionsgrößen als primäre Endpunkte akzeptiert werden [18-20]. In beiden Studienarmen unterschieden sich die unerwünschten Wirkungen nicht signifikant. Die Verträglichkeit der Therapie war sehr gut. In der NACGruppe wurde eine signifikant geringere Knochenmarkstoxizitätsrate festgestellt, was möglicherweise auf einem protektiven Effekt von NAC gegenüber myelotoxischen Effekten von Azathioprin beruht. Bei Patienten mit einer IPF findet sich ein Exzess an reaktiven Sauerstoffspezies (ROS) und ein Mangel an Antioxidantien, insbesondere Glutathion. NAC dient hierbei als Cysteinlieferant für die Glutathionsynthese. [21,22]. Das Präparat NAC ist aufgrund des Therapiegewinns und des günstigen Nebenwirkungsprofils sowie der vergleichsweise niedrigen Kosten empfehlenswert. 




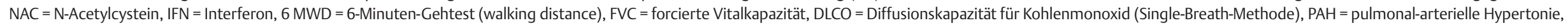
$\mathrm{UIP}=$ ususal interstitial pneumonitis, mPAP = mittlerer pulmonal-arterieller Druck. NCT-Nummern von der Federal Drug Administration (www.clinicaltrails.gov/ct2/results?cond=\%22ldiopathic+Pulmonary+Fibrosis\%22).

\begin{tabular}{|c|c|c|c|c|c|}
\hline Studie & Prüfmedikation & Studiendauer/-design & Patientenzahl & $\begin{array}{l}\text { Besonderheiten der } \\
\text { Einschlusskriterien }\end{array}$ & Primärer Studienendpunkt \\
\hline $\begin{array}{l}\text { IFIGENIA } \\
{[10]}\end{array}$ & $\begin{array}{l}\text { NAC }(3 \times 600 \mathrm{mg} / \text { Tag oral, } 1 \text { Jahr }) \text { vs. } \\
\text { Placebo zzgl. Prednisolon/Azathioprin }\end{array}$ & 3 Jahre $(03 / 2000-07 / 2003)$ & 184 & $\begin{array}{l}\text { IPF nach Kriterien American } \\
\text { Thoracic Society und European } \\
\text { Respiratory Society }\end{array}$ & $\triangle \mathrm{VC}$ und $\mathrm{DLCO}$ \\
\hline $\begin{array}{l}\text { GIPF-001 } \\
{[23]}\end{array}$ & $\begin{array}{l}\text { IFN- }-1 / \beta \text { subkutan }(200 \mu \mathrm{g}, 3 \times / \text { Woche, } \\
\text { Phase III) vs. Placebo }\end{array}$ & 2,5 Jahre $(04 / 2000-12 / 2002)$ & 330 & $\begin{array}{l}\text { IPF, Steroid-Non-Responder, } \\
>3 \text { Jahre }\end{array}$ & Progressionsfreies Überleben \\
\hline GIPF-001 (NCT00047658) & $\begin{array}{l}\text { IFN- }-1 \beta \text { subkutan }(200 \mu \mathrm{g}, 3 \times \text { / Woche, } \\
\text { Phase III) vs. Placebo }\end{array}$ & 1,5 Jahre $(11 / 2001-05 / 2003)$ & 32 & $\begin{array}{l}\text { IPF, Steroid-Non-Responder, } \\
>3 \text { Jahre }\end{array}$ & $\triangle \mathrm{FVC}$ \\
\hline $\begin{array}{l}\text { GIPF-001 extension } \\
\text { (NCT00052052) }\end{array}$ & $\begin{array}{l}\text { IFN- }-1 \beta \text { subkutan }(200 \mu \mathrm{g}, 3 \times / \text { Woche, } \\
\text { für } 48 \text { Wochen, Phase III) vs. Placebo }\end{array}$ & $\begin{array}{l}2 \text { Jahre (09/2002-09/2004), } \\
\text { offen, ein Arm, Beobachtung }\end{array}$ & 210 & IPF, wie andere GIPF-Studien & $\begin{array}{l}\text { Sicherheitsaspekte, Überleben, } \\
\text { anti-IFN- } \gamma-1 \beta \text { Antikörper }\end{array}$ \\
\hline $\begin{array}{l}\text { CIPF-002/GIPF-004 extension } \\
\text { (NCT00076635) }\end{array}$ & $\begin{array}{l}\text { IFN- } \gamma-1 \beta \text { subkutan }(200 \mu \mathrm{g}, 3 \times / \text { Woche, } \\
\text { Phase III) vs. Placebo }\end{array}$ & $\begin{array}{l}5 \text { Jahre }(11 / 2003-04 / 2007 \text {, } \\
\text { vorzeitig abgebrochen), offen, } \\
\text { ein Arm, Beobachtung }\end{array}$ & 91 & IPF, wie andere GIPF-Studien & $\begin{array}{l}\text { Sicherheitsaspekte, Überleben, } \\
\text { anti-IFN- } \gamma-1 \beta \text { Antikörper }\end{array}$ \\
\hline INSPIRE (NCT00075998) & $\begin{array}{l}\text { IFN- } \gamma \text {-1 } \beta \text { subkutan }(200 \mu \mathrm{g}, 3 \times / \text { Woche, } \\
2 \text { Jahre Therapie, Phase III) vs. Placebo }\end{array}$ & 3,5 Jahre $(12 / 2003-05 / 2007)$ & 826 & $\begin{array}{l}\text { FVC } \geq 55 \% \text { Sollwert, DLCO } \geq 35 \% \\
\text { Sollwert, Erkrankungsprogress, } \\
6 \mathrm{MWD} \geq 150 \mathrm{~m}\end{array}$ & Progressionsfreies Überleben \\
\hline $\begin{array}{l}\text { Antikoagulation } \\
\text { [15] }\end{array}$ & $\begin{array}{l}\text { Warfarin }(75 \mathrm{IU} / \mathrm{kg} / \mathrm{Tag}) \text { oder Heparin }{ }^{+} \\
\text {zzgl. Prednisolon vs. Prednisolon } \\
\text { (10 mg-20 mg/Tag) allein }\end{array}$ & $\begin{array}{l}3 \text { Jahre }(04 / 2001-04 / 2004) \text {, } \\
\text { Beobachtungszeit } 35 \text { - } 1106 \text { Tage, } \\
\text { Median } 399 \text { Tage) }\end{array}$ & 56 & $\begin{array}{l}\text { UIP in Biopsie oder IPF nach HRCT- } \\
\text { Kriterien }\end{array}$ & Überleben, krankenhausfreie Zeit \\
\hline $\begin{array}{l}\text { Pirfenidon } \\
\text { [11] }\end{array}$ & $\begin{array}{l}\text { Pirfenidon (Dosiseskalation } 200 \mathrm{mg} / 2 \times / \text { Tag } \\
\text { bis } 600 \mathrm{mg} / 3 \times / \text { Tag, } 9 \text { Monate) vs. } \\
\text { Placebo. Mit/ohne Prednisolon } \leq 10 \mathrm{mg} / \text { Tag }\end{array}$ & 1 Jahr & 107 & & SaO2-Abfall im 6 MWD \\
\hline $\begin{array}{l}\text { Etanercept } \\
{[12]}\end{array}$ & $\begin{array}{l}\text { Etanercept ( } 25 \text { mg s. c./Tag für } 1 \text { Jahr, Phase } \\
\text { II) vs. Placebo }\end{array}$ & 3 Jahre $(07 / 2003-05 / 2006)$ & 87 & $\begin{array}{l}\mathrm{FVC}>45 \% \mathrm{~d} \text {. Sollwertes, } \\
\mathrm{DLCO}>25 \% \text { des Sollwertes, } \\
\mathrm{PaO} 2>55 \mathrm{~mm} \mathrm{Hg} / \mathrm{SaO} 2>88 \%\end{array}$ & $\begin{array}{l}\text { FEV\% des Sollwertes, DLCO des } \\
\text { Sollwertes }\end{array}$ \\
\hline $\begin{array}{l}\text { BUILD-1 } \\
{[14]}\end{array}$ & Bosentan $(2 \times 125 \mathrm{mg} /$ Tag oral) vs. Placebo & $\begin{array}{l}1 \text { Jahr }(08 / 2003-11 / 2005) \text { mit } \\
\text { Fortsetzung im offenen Studien- } \\
\text { design (siehe } \square \text { Tab. } 2 \text { ) }\end{array}$ & 158 & $\begin{array}{l}\text { Krankheitsdauer }<3 \text { Jahre, } 6 \text { MWD, } \\
\text { FVC }>50 \% \text { Sollwert }(<1,2 \text { I) oder } \\
\text { DLCO }>30 \% \text { Sollwert oder } \\
\text { RV } \geq 120 \% \text { Sollwert }\end{array}$ & $\triangle 6 M W D$ \\
\hline $\begin{array}{l}\text { FG-3019 } \\
\text { (NCT00074698) }\end{array}$ & FG-3019 (anti-CTGF-Antikörper, Phase I) & $\begin{array}{l}1 \text { Jahr }(12 / 2003-05 / 2004) \\
\text { Offen, nicht-randomisiert, nicht } \\
\text { placebokontrolliert }\end{array}$ & 27 & $\begin{array}{l}\text { mittels offener Lungenbiopsie } \\
\text { nachgewiesener IPF }\end{array}$ & Sicherheitsaspekte \\
\hline $\begin{array}{l}\text { Mesylate } \\
\text { (NCT00131274) }\end{array}$ & $\begin{array}{l}\text { Imatinib Mesylate }(600 \mathrm{mg} / \text { Tag über } \\
2 \text { Jahre) vs. Placebo }\end{array}$ & 4 Jahre $(04 / 2003-08 / 2007)$ & 120 & $\begin{array}{l}>10 \% \text { FFVC-Abfall, Verschlimme- } \\
\text { rung im RÖ-Thoraxbild, FVC > } 55 \% \\
\text { Sollwert, DLCO > } 35 \% \text { Sollwert, } \\
\text { PaO2 > } 55-60 \mathrm{~mm} \mathrm{Hg}\end{array}$ & $\Delta \mathrm{FVC}>10 \%$ oder Tod \\
\hline $\begin{array}{l}\text { FIBROSAND } \\
\text { (NCT00463983 }\end{array}$ & $\begin{array}{l}\text { Octreotide ( } 30 \mathrm{mg} \text { intramuskulär alle } \\
4 \text { Wochen über } 48 \text { Wochen; Phase I+ II) }\end{array}$ & 2 Jahre (10/2006 bis $08 / 2008)$ & 25 & $\begin{array}{l}\text { IPF nach Kriterien American Thora- } \\
\text { cic Society und European Respira- } \\
\text { tory Society }\end{array}$ & $\triangle \mathrm{FVC}$ und $\mathrm{DLCO}$ \\
\hline
\end{tabular}




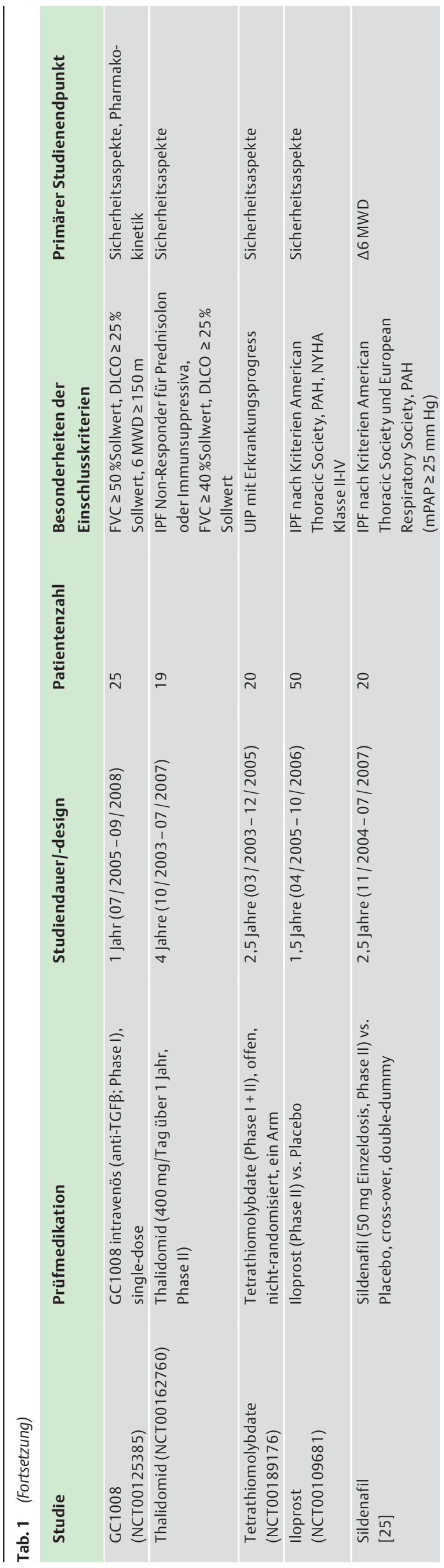

\section{Interferon-gamma \\ 1. GIPF-001-Studie}

In dieser Studie wurde die klinische Wirksamkeit von IFN-gamma evaluiert. Dazu wurden randomisiert, doppelblind und placebokontrolliert 330 Patienten mit einer IPF eingeschlossen (৫ Tab. 1). Primärer Endpunkt war die Verlängerung des progressionsfreien Überlebens. Im Mittel wurden die Patienten 58 Wochen therapiert und es zeigte sich ein Trend zur Verbesserung des progressionsfreien Überlebens, wobei das Signifikanzniveau nicht erreicht wurde. In der Subgruppenanalyse zeigte sich dann, dass im Gegensatz zum Gesamtkollektiv diejenigen Patienten mit einer geringeren Lungenfunktionseinschränkung und Erkrankungsschwere eine signifikante Verbesserung der Überlebenszeit aufwiesen (4\% und $12 \%$ Mortalität im Vergleich, $p=0,04$ ). Diverse sekundäre Parameter, wie z.B. die Lungenfunktion, die Blutgasanalyse oder der Lebensqualitäts-Score, unterschieden sich zwischen den Behandlungsgruppen allerdings nicht [23]. Eine griechische Studie belegte, dass die FVC in der IFN-gamma-Gruppe höher lag als in der zum Vergleich dienenden Colchicin-Gruppe und die Mortalität niedriger (15,6\% vs. 38,8\%) [8].

\section{GIPF-006 (INSPIRE-Studie)}

Primärer Endpunkt der INSPIRE-Studie, in der die Patienten über einen Zeitraum von zwei Jahren inhalatives IFN-gamma $1 \beta$ erhielten, war die Überlebenszeit. Diese Studie wurde vorzeitig nach erfolgter Interimsanalyse gestoppt. Der Grund lag im fehlenden Wirksamkeitsnachweis der Substanz im Vergleich zu Placebo, sodass davon ausgegangen werden konnte, dass auch eine Fortführung der Therapie bis zum geplanten Studienende keinen Benefit gebracht hätte. Die Grundlage der Interferontherapie bildet die Modulation der Th1/T2 Balance und deren direkten antifibrotischen Eigenschaften. Durch die im Vergleich zu anderen Studien inkonsistenten Ergebnisse und der erheblichen Nebenwirkungen (Fieber, grippeähnliche Symptome nach Injektion sowie Fälle einer respiratorischen Insuffizienz) ist eine IFN-gamma Therapie weder mittels inhalativer noch subkutaner Applikation zu empfehlen [9].

\section{Antikoagulantien (Warfarin oder niedermolekulares Heparin)}

Einen anderen Therapieansatz verfolgten Kubo et al. [15]. In dieser Studie wurden 56 Patienten, die wegen einer IPF mit Prednisolon therapiert wurden, zusätzlich antikoagulativ (entweder mit Warfarin oder mit niedermolekularem Heparin) behandelt. Die Überlebenskurven unterschieden sich zugunsten der Antikoagulantientherapie mit einer Senkung des Mortalitätsrisikos $(p=0,04)$. Die Senkung der Mortalität in der Verumgruppe war mit einer Senkung der akuten IPF-Exazerbation assoziiert (18\% vs. $71 \%, p=0,008$ ). Die Zeit krankenhausfreier Tage war in beiden Gruppen gleich [15]. Allerdings wies diese Studie einige methodische Schwächen auf (keine Verblindung, unterschiedliche Häufigkeit von Therapieabbrüchen, keine Intention zur number to treat-Analyse, mehr als ein Viertel der Patienten der Antikoagulanzgruppe schieden schon vor Behandlungsbeginn aus der Studie aus, kein sicherer Ausschluss einer Lungenembolie als mögliche Ursache der Verschlechterung).

\section{Pirfenidon}

Pirfenidon ist ein Pyridonderivat, das in vitro die Synthese proinflammatorischer Zytokine (z. B. TNF alpha, IL6) sowie die TGF$\beta$-induzierte Kollagensynthese und Fibroblastenproliferation hemmt. Die antientzündliche Wirkung dieser Substanz hat dazu 




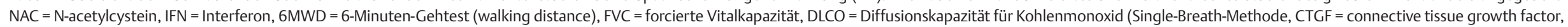

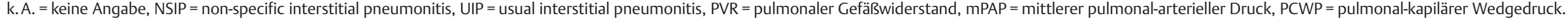
NCT-Nummern von der Federal Drug Administration (www.clinicaltrails.gov/ct2/results?cond=\%22ldiopathic+Pulmonary+Fibrosis\%22).

\begin{tabular}{|c|c|c|c|c|c|}
\hline Studie/FDA-Nr. & Prüfmedikation & Studiendauer/-design & Patientenzahl & $\begin{array}{l}\text { Besonderheiten der } \\
\text { Einschlusskriterien }\end{array}$ & Primärer Studienendpunkt \\
\hline $\begin{array}{l}\text { Prednisolon/Azathioprin } \\
\text { (NCT00518310) }\end{array}$ & $\begin{array}{l}\text { Prednisolon } 0,5 \mathrm{mg} / \mathrm{kg} / \text { Tag } 4 \text { Wochen, } \\
0,25 \mathrm{mg} / \mathrm{kg} / \mathrm{Tag} 8 \text { Wochen, gefolgt von } \\
0,25-0,125 \mathrm{mg} / \mathrm{kg} / \mathrm{Tag} \\
\text { Azathioprin } 2-3 \mathrm{mg} / \mathrm{kg} / \text { Tag, vs Placebo }\end{array}$ & $\begin{array}{l}2 \text { Jahre (geplant: } 05 \text { / } 2005 \text { bis } \\
12 / 2008 \text { ) } \\
\text { Randomisiert und placebo- } \\
\text { kontrolliert }\end{array}$ & 100 & $\begin{array}{l}\text { IPF } \geq 3 \text { Monate, FVC } 50-90 \% \text { Sollwert, } \\
\text { DLCO } \geq 35 \% \text {, PaO2 } \geq 55 \mathrm{~mm} \mathrm{Hg} \text {, HRCT } \\
\text { mit IPF-Kriterien }\end{array}$ & progressionsfreies Überleben \\
\hline $\begin{array}{l}\text { Thalidomid } \\
\text { (NCT00600028) }\end{array}$ & $\begin{array}{l}\text { Thalidomid ( } 50 \text { - } 100 \text { mg, } 6 \text { Monate, } \\
\text { Phase III) vs. Placebo }\end{array}$ & 2 Jahre $(12 / 2007-12 / 2009)$ & 20 & $\begin{array}{l}\text { FVC }>40 \%-<90 \% \text { Sollwert, TLC }> \\
40 \%-80 \% \text { Sollwert, DLCO }>30-<90 \% \\
\text { Sollwert, chronischer Husten }\end{array}$ & $\begin{array}{l}\text { Hustenreduktion } \\
\text { (nach } 6 \text { Therapiemonaten) }\end{array}$ \\
\hline Sildenafil (NCT00625079) & $\begin{array}{l}\text { Sildenafil }(3 \times 20 \mathrm{mg} / \text { Tag, Phase IV) vs. Pla- } \\
\text { cebo oder vs. Anderer PAH-Senker, nur Beob- } \\
\text { achtung bei PAH-freie Beobachtungsgruppe }\end{array}$ & $\begin{array}{l}3 \text { Jahre }(02 / 2007-12 / 2009) \text {, } \\
\text { single center }\end{array}$ & 50 & $\begin{array}{l}\text { IPF-Patienten, die für die Lungen- } \\
\text { transplantation gelistet sind, } \\
\text { MWD } \geq 50 \text { Meter }\end{array}$ & $\triangle 6 \mathrm{MWD}$ \\
\hline $\begin{array}{l}\text { Minocyclin } \\
\text { (NCT00203697) }\end{array}$ & $\begin{array}{l}\text { Minocyclin (Phase III) zzgl. zu Prednisolon } \\
<0,3 \mathrm{mg} / \mathrm{kg} \text { und Azathoprin oder Cyclo- } \\
\text { phosphamid }\end{array}$ & $\begin{array}{l}\text { randomisiert, placebokontrolliert. } \\
\text { Studie noch aktiv, aber derzeit kei- } \\
\text { ne Patientenrekrutierung }\end{array}$ & k. A. & $\begin{array}{l}\text { IPF }>3 \text { Monate, FVC }>40 \%-<90 \% \text { Soll- } \\
\text { wert, } \mathrm{TLC}>40 \%-80 \% \text { Sollwert, } \\
\text { DLCO }>20 \% \text { Sollwert, PaO2 }>50 \mathrm{~mm} \mathrm{Hg}\end{array}$ & k. A. \\
\hline $\begin{array}{l}\text { Bosentan (Build3 extension; } \\
\text { NCT0631475) }\end{array}$ & $\begin{array}{l}\text { Bosentan ( } 62,5 \text { oder } 125 \mathrm{mg} / \text { Tag oral, } \\
\text { Phase 3) }\end{array}$ & $\begin{array}{l}1 \mathrm{Jahr}(03 / 2008-03 / 2009) \text { ein } \\
\text { Arm in der Nachbeobachtung }\end{array}$ & 600 & $\begin{array}{l}\text { Krankheitsdauer }<3 \text { Jahre, } 6 \text { MWD, } \\
\text { FVC }<50 \% \text { Sollwert }(<1,2 \text { I) oder } \\
\text { DLCO }<30 \% \text { Sollwert oder RV } \geq 120 \% \\
\text { Sollwert }\end{array}$ & Sicherheitsaspekte \\
\hline $\begin{array}{l}\text { Pirfenidon (PIPF-004 + PIPF } \\
006 \text { extension, NCT0062038) }\end{array}$ & Pirfenidon ((2403 mg/Tag, über 72 Wochen) & $\begin{array}{l}3,5 \text { Jahre }(08 / 2008-05 / 2011) \text {, } \\
\text { offen, ein Arm in der Nachbeob- } \\
\text { achtung }\end{array}$ & 750 & $\begin{array}{l}\text { FVC } \geq 50 \% \text { Sollwert, DLCO } \geq 35 \% \text { Soll- } \\
\text { wert, } 6 \mathrm{MWD} \geq 150 \mathrm{~m}(\mathrm{SaO} 2 \geq 83 \%) \\
\text { Compliancerate } \geq 80 \%\end{array}$ & $\begin{array}{l}\text { Sicherheitsaspekte, uner- } \\
\text { wünschte Nebenwirkungen }\end{array}$ \\
\hline Treprostinil (NCT00703339) & $\begin{array}{l}\text { Treprostinil inhalativ (single dose escalating, } \\
\text { Phase II) }\end{array}$ & 1,5 Jahre $(06 / 2008-12 / 2009)$ & 16 & $\begin{array}{l}\text { FVC } \geq 30 \%-70 \% \text { Sollwert, DLCO } \geq \\
20 \%-70 \% \text { Sollwert }\end{array}$ & Sicherheitsaspekte \\
\hline Treprostinil (NCT00705133) & $\begin{array}{l}\text { Treprostinil inhalativ oder subkutan } \\
\text { (1 } \mathrm{ng} / \mathrm{kg} / \text { Minute/Tag bis } 40 \mathrm{ng} / \mathrm{kg} / \text { Minute) }\end{array}$ & $\begin{array}{l}\text { 1,5 Jahre (01 / } 2008-02 \text { / 2009), } \\
\text { offen, ein Arm }\end{array}$ & 20 & $\begin{array}{l}\text { UIP (Biopsie), mPAP }>35 \mathrm{~mm} \mathrm{GH} \text { und } \\
\text { PVR }>3 \text { woods-units und } \\
\text { PCWP }<18 \mathrm{~mm} \mathrm{Hg}\end{array}$ & $\triangle 6 \mathrm{MWD}$ \\
\hline $\begin{array}{l}\text { Zileutin } \\
\text { (NCT00262405) }\end{array}$ & $\begin{array}{l}\text { Zileutin zzgl. Prednisolon mit oder ohne } \\
\text { Azathioprin }\end{array}$ & $\begin{array}{l}\text { k. A. (Start 01/2001), randomi- } \\
\text { siert, placebokontrolliert. Studie } \\
\text { noch aktiv, aber derzeit keine Pa- } \\
\text { tientenrekrutierung }\end{array}$ & 140 & IPF & k. A. \\
\hline Bosentan (NCT00625468) & $\begin{array}{l}\text { Bosentan }(62,5-125 \mathrm{mg} / 2 \times / \text { Tag, Phase IV), } \\
\text { offen, randomisiert, placebokontrolliert }\end{array}$ & $\begin{array}{l}10 / 2 \text { Jahre }(10 / 2007-12 / 2009) \text {, } \\
\text { single center }\end{array}$ & 50 & $\begin{array}{l}\text { IPF mit PAH, die für Lungentransplanta- } \\
\text { tion vorgesehen sind, } 6 \mathrm{MWD} \geq 50 \mathrm{~m}\end{array}$ & $\triangle 6 \mathrm{MWD}$ \\
\hline BUILD-3 (NCT00391443) & $\begin{array}{l}\text { Bosentan }(2 \times 62,5 \text { bis } 2 \times 125 \mathrm{mg} / \text { Tag oral, } \\
\text { Phase III) vs. Placebo }\end{array}$ & $\begin{array}{l}3 \text { Jahre (11/ } 2006-12 \text { / 2009), } \\
\text { Studie noch aktiv, aber derzeit } \\
\text { keine Patientenrekrutierung }\end{array}$ & 600 & $\begin{array}{l}\text { IPF nach Kriterien American Thoracic } \\
\text { Society und European Respiratory } \\
\text { Society, Krankheitsdauer < } 3 \text { Jahre }\end{array}$ & $\begin{array}{l}\text { Zeit bis Verschlechterung } \\
\text { der Erkrankung oder Tod }\end{array}$ \\
\hline $\begin{array}{l}\text { Artemis } \\
\text { (NCT00768300) }\end{array}$ & $\begin{array}{l}\text { Ambrisentan ( } 5 \mathrm{mg} / \text { Tag oder } 10 \mathrm{mg} / \mathrm{Tag} \text {, } \\
\text { Phase III) vs. Placebo }\end{array}$ & 4 Jahre $(12 / 2008-12 / 2012)$ & 600 & IPF $\geq 3$ Jahre, Honigwaben im HRCT & $\begin{array}{l}\text { Zeit bis Verschlechterung } \\
\text { der Erkrankung oder Tod }\end{array}$ \\
\hline $\begin{array}{l}\text { Bosentan } \\
\text { (NCT00637065) }\end{array}$ & $\begin{array}{l}\text { Bosentan }(62,5 \mathrm{mg} / 2 \times / \text { Tag bis } 125 \mathrm{mg} / \\
2 \times / \text { Tag, } 16 \text { Wochen, Phase IV) vs. Placebo }\end{array}$ & 3 Jahre $(04 / 2008-08 / 2010)$ & 48 & $\begin{array}{l}\text { IPF und NSIP nach Kriterien American } \\
\text { Thoracic Society und European } \\
\text { Respiratory Society }\end{array}$ & $\begin{array}{l}\Delta \text { pulmonal-arterieller } \\
\text { Widerstand }\end{array}$ \\
\hline
\end{tabular}




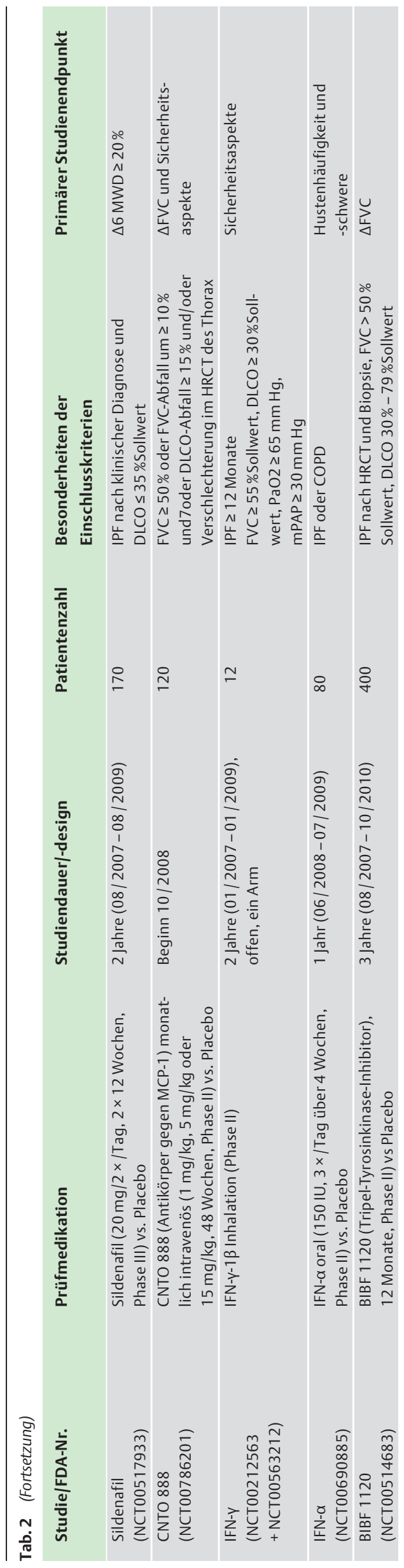

geführt, dass Pirfenidon in mehreren IPF-Therapiestudien eingesetzt wurde. Die von Azuma et al. 2005 publizierte PirfenidonStudie verfehlte allerdings den primären Endpunkt (Reduktion des Sauerstoffsättigungsabfalls im 6-Minuten-Gehtest) [11]. Diese Studie wurde vorzeitig abgebrochen, da es in dem Placeboarm zu einer Häufung von akuten Exazerbationen kam: 5 akute Exazerbationen bei 35 Patienten gegenüber keinem Fall im Verumarm [11]. Trotz dieses vorzeitigen Studienabbruchs zeigte sich ein grenzwertig signifikanter Effekt von Pirfenidon auf den primären Studienendpunkt - minimale arterielle Sauerstoffsättigung während eines modifizierten 6-Minuten-Gehtests. Außerdem wurde ein signifikant geringerer Abfall der VC in der Verumgruppe, verglichen zu Placebo, nach 9 Monaten festgestellt. Als wesentliche Nebenwirkung ist die hohe Rate der aufgetretenen Phototoxizität zu erwähnen (bis zu 50\% d.F.).

\section{Etanercept}

Etanercept ist ein gentechnologisch hergestelltes Fusionsprotein. Es besitzt die Ligandenbindungsdomäne des humanen TNF-Rezeptors-2 und ist deshalb in der Lage, TNF-alpha und TNF-beta zu binden. Auch hier verfehlte der Einsatz dieser Substanz den primären Studienendpunkt [12]. Nach einer 12-monatigen Therapie errechnete sich zwischen den beiden Therapiegruppen kein signifikanter Vorteil für Etanercept in Bezug auf die Änderung der VC, der DLCO und dem alveolären Sauerstoffgradienten. Es ergab sich lediglich ein Trend zur Reduktion der Erkrankungsprogression anhand verschiedener Sekundärparameter [12].

\section{Bosentan \\ BUILD-1-Studie}

In der BUILD-1-Studie erhielten 158 gut charakterisierte IPF-Patienten den dualen Endothelin-Rezeptorantagonisten Bosentan. Schwer kranke Patienten oder Patienten im Endstadium sowie die mit einer schweren PAH wurden ausgeschlossen. Der Therapiezeitraum wurde auf 12 Monate festgelegt. 49 Patienten (25 in der Bosentan- und 24 in der Placebogruppe) schieden wegen einer Verschlechterung der IPF vorzeitig aus dieser Studie aus [14]. Bei den noch in der Studie verbliebenen Patienten zeigte sich im primären Endpunkt 6-Minutengehstrecke kein signifikanter Gruppenunterschied. Im Trend war eine Verbesserung der sekundären Endparameter Erkrankungsprogression, Verlangsammung der Verschlechterung der Lungenfunktion und Verminderung der akuten Exazerbationen zu erkennen. Die respiratorisch unerwünschten Nebeneffekte lagen in der Bosentan-Gruppe niedriger (17,6 vs. $27,4 \%$ ). Gleiches gilt für die Angabe von Dyspnoe (13,5 vs. $19 \%)$ und akuter Verschlechterung der IPF (16,2 vs. $23,8 \%$ ). Am meisten profitierten offenbar die Subgruppen von solchen Patienten, die im HRCT-Bild nur wenige Honigwaben gezeigt hatten und deren IPF per Biopsie histologisch nachgewiesen wurde. Ungeachtet der positiven Trends und vielversprechenden Subgruppenanalysen verfehlte auch die BUILD-1-Studie den Nachweis einer klinischen Effektivität von Bosentan [14]. Ob Bosentan bei einer histologisch definierten IPF und im HRCT geringen Nachweis von Honigwaben ( $<5 \%$ ), d. h. bei strenger selektionierten Patienten, einen besseren Effekt aufweist, wird derzeit in der BUILD-3 Studie untersucht [24]. 
Fazit

In Zusammenschau der Ergebnisse der hier vorgestellten Studien hat lediglich die IFIGENIA-Studie den primären Endpunkt (signifikante Verminderung des Verlusts an Vitalkapazität und Diffussionskapazität) erreicht. Aufgrund dieser Tatsache und des geringen Nebenwirkungsprofils kann hochdosiertes NAC $(3 \times 600 \mathrm{mg} /$ die p.o.) zusätzlich zu Prednisolon und Azathioprin empfohlen werden.

Für Interferon-gamma konnte kein Therapieeffekt gezeigt werden, sodass IFN-gamma zur Therapie der IPF nicht empfohlen werden kann. Darüber hinaus gibt es zum jetzigen Zeitpunkt weder für Pirfenidon noch den Endothelin-Rezeptor-Antagonisten Bosentan eine Therapieindikation außerhalb von Studien. Der Stellenwert der oralen Antikoagulation bei Patienten mit einer IPF mit oder ohne Vorliegen einer pulmonalen Hypertonie ist bei fehlendem Nachweis einer zusätzlichen Lungenembolie noch unklar und kann gegenwärtig nicht empfohlen werden.

Die hier zitierten Studien zeigen auf der einen Seite, dass in Subgruppen durchaus ein Vorteil der evaluierten Pharmaka erzielbar ist. Andererseits zeigen die Studien auch, wie schwierig es ist, IPF-Patienten erfolgreich zu therapieren. Die erzielbaren Veränderungen sind gering und die Durchführung von klinischen Studien sind z.T. problematisch aufgrund des Auftretens von nicht vorhersehbaren Variationen im Krankheitsverlauf. Die in der - Tab. 2 aufgelisteten, zur Zeit durchgeführten Studien lassen aber hoffen, dass auch in Zukunft Ergebnisse generiert werden, die unser Verständnis für die Erkrankung intensivieren, woraus sich letztendlich vielleicht auch eine Therapie definieren lässt, mit der wir diese sehr schwere Erkrankung in Zukunft besser therapieren können.

\section{Interessenkonflikte}

Prof. Dr. A. Gillissen hat in den letzten fünf Jahren Vortragshonorare, Honorare für eine Beratertätigkeit und Reisekostenunterstützungen erhalten von: GlaxoSmithkline, Boehringer Ingelheim, Talecris, AstraZeneca, Pfizer, Novartis, Janssen-Cilag. Zudem wurde/wird er über die Robert-Koch-Klinik, Thoraxzentrum des Klinikums St. Georg, Leipzig, bei Forschungsvorhaben im gleichen Zeitraum unterstützt von: Pierre Fabre, Roche, Boehringer Ingelheim, AstraZeneca, MEDA Pharma, Lilly, Novartis, Johnson \& Johnson, Sanofi-Aventis.

Dr. med. K. Gutjahr hat in den letzten fünf Jahren Reisekostenunterstützung von Actelion erhalten.

\section{Literatur}

1 Demedts M, Wells AU, Antó JM et al. Interstitial lung disease: an epidemiological overview. Eur Respir J 2001; 18: 2s-16s

2 Raghu G, Weycker D, Edelsberg J. Incidence and prevalence of idiopathic pulmonary fibrosis. Am J Respir Crit Care Med 2006; 174: 810- 816

3 Martinez FJ, Safrin S, Weycker D et al. The clinical course of patients with idiopathic pulmonary fibrosis. Ann Intern Med 2005; 142: 963 967

4 Ziegenhagen MW, Müller-Quernheim J. Idiopathische pulmonale Fibrose (IPF): Diagnose, Differenzialdiagnose und neue Therapieoptionen. Dtsch Med Wschr 2004; 129: 494-498

5 Noth I, Martinez FJ. Recent advances in idiopathic pulmonary fibrosis. Chest 2007; 132: 637-650

6 Günther A, Markart P, Eickelberg O, Seeger W. Lungenfibrosen - ein therapeutisches Dilemma? Med Klinik 2006; 101: 308-312

7 Tzortzaki EG, Antoniou KM, Zervou MI et al. Effects of antifibrotic agents on TGF-beta1, CTGF and IFN-gamma expression in patients with idioapathic pulmonary fibrosis. Respir Med 2007; 101: 1821 - 1829

8 Antoniou KM, Nicholson AG, Dimadi M et al. Long-term clinical effects of interferon gamma- $1 \beta$ and colchicine in idiopathic pulmonary fibrosis. Eur Respir J 2006; 28: 496 - 504

9 Pacanowski MA, Amsden GW. Interferon gamma-1B in the treatment of idiopathic pulmonary fibrosis. Ann Pharmacother 2005; 39: 1678 1686

10 Demedts M, Behr J, Buhl R et al. High-dose acetylcysteine in idiopathic pulmonary fibrosis. N Engl J Med 2005; 353: 2229-2242

11 Azuma A, Nukiwa T, Tsuboi E et al. Double-blind, placebo-controlled trial of pirfenidone in patients with idiopathic pulmonary fibrosis. Am J Respir Crit Care Med 2005; 171: 1040-1047

12 Raghu G, Brown KK, Costabel $U$ et al. A randomized placebo controlled trial assessing the efficacy and safety of etanercept in patients with idiopathic pulmonary fibrosis (IPF). Am J Respir Crit Care Med 2008; 178: 948 - 955

13 Antoniu SA. Targeting the endothelin pathway in the idiopathic pulmonary fibrosis: the role of bosentan. Expert Opin Ther Targets 2008; 12: $1077-1084$

14 King TE Jr., Behr J, Brown KK et al. BUILD-1: a randomized placebo-controlled trial of bosentan in idiopathic pulmonary fibrosis. Am J Respir Crit Care Med 2008; 177: 75-81

15 Kubo H, Nakayama K, Yanai M et al. Anticoagulant therapy for idiopathic pulmonary fibrosis. Chest 2005; 128: $1475-1482$

16 Holland A, Hill C. Physical training for interstitial lung disease. Cochrane Database Syst Rev 2008; 4: CD006322

17 Brown KK, Wells $A U$. Recent clinical trials in idiopathic pulmonary fibrosis and the BUILD-1 study. Eur Respir Rev 2008; 17: 116-122

18 Flaherty KR, Thwaite EL, Kazerooni EA et al. Radiological versus histological diagnosis in UIP and NSIP: survival implications. Thorax 2003; 58: $143-148$

19 Collard HR, King TE Jr., Bartelson BB et al. Changes in clincial and physiologic variables predict survival in idiopathic pulmonary fibrosis. Am J Respir Crit Care Med 2003; 168: 538- 542

20 Latsi P, du Bois RM, Nicholson AG et al. Fibrotic idiopathic interstitial pneumonia: the prognostic value of longitudinal functional trends. Am J Respir Crit Care Med 2003; 168: 531-537

21 Gillissen A, Nowak D. Characterization of N-acetylcyteine and ambroxol in antioxidant therapy. Resp Med 1998; 92: 609-623

22 Behr J, Maier K, Degenkolb B et al. Antioxidative and clinical effects of high-dose $\mathrm{N}$-acetylcysteine in fibrosing alveolitis. Adjunctive therapy to maintenance immunosuppression. Am J Respir Crit Care Med 1997; 156: $1897-1901$

23 Raghu G, Brown KK, Bradford WZ et al. A placebo controlled trial of interferon gamma- $1 \beta$ in patients with idiopathic pulmonary fibrosis. $\mathrm{N}$ Engl J Med 2004; 350: 125-133

24 King TE Jr. Bosentan for idiopathic pulmonary fibrosis. Curr Opin Investig Drugs 2008; 9: 1171-1179

25 Collard HR, Anstrom KJ, Schwarz MI, Zisman DA. Sildenafil improves walk distance in idiopathic pulmonary fibrosis. Chest 2007; 131: 897-899 